\title{
Crystal locomotion driven by photo-triggered phase transition
}

\author{
Y. Hagiwara ${ }^{1}$, A. Takanabe ${ }^{1}$, T. Asahi, ${ }^{1,2}$, H. Koshima ${ }^{2}$ \\ ${ }^{1}$ Graduate school of Advanced Science and Engineering, Waseda University, 3-4-1 Okubo, Shinjuku-ku, Tokyo, Japan, \\ ${ }^{2}$ Research Organization for Nano \& Life Innovation, Waseda University, 513 Waseda-tsurumakicho, Shinjuku-ku, Tokyo, Japan.
}

$$
\text { miler-yh0514@fuji.waseda.jp }
$$

Mechanical crystals are expected to be applicable for actuators and soft robots [1]. Before the past decade, we have developed many mechanical crystals based on photoisomerization [2], and some based on phase transition [3] and photothermal effect [4]. In 2019, we have found a new kind of phase transitions, referred to as the photo-triggered phase transition [5]. The photochromic crystal exhibiting a thermal, reversible single-crystal-to-single-crystal phase transition upon heating and cooling, transform to the identical phase upon light irradiation at temperatures lower than thermal phase transition temperature. A chiral salicylidnephenylethylamine [enol- $(S)$-1] crystal is known to undergo photoisomerization (Fig. 1a) [6], and thermal phase transition [7]. We have found that the enol-(S)-1 crystal exhibited the photo-triggered phase transition.

Upon heating, the enol- $(S)-1$ crystal in the a-phase $\left(P 2_{1}\right)$ transformed to the $\mathrm{b}$-phase $\left(P 2{ }_{1} 2_{1} 2_{1}\right)$ with the discontinuous $\mathrm{b}$-angle change to $90^{\circ}$ at $0^{\circ} \mathrm{C}$ due to thermal phase transition from monoclinic to orthorhombic crystal system (yellow circles, Fig. 1b). Under UV light $(365 \mathrm{~nm})$ irradiation, the a-phase changed to the b-phase even at $-30{ }^{\circ} \mathrm{C}$ (orange circles, Fig. $1 \mathrm{~b}$ ). The mechanism was revealed that the photo-triggered phase transition is driven by the strain near the irradiated surface produced by the photoisomerization. A thick crystal in the a-phase deformed by the photo-triggered phase transition to the b-phase upon UV light irradiation; the surface temperature did not reach the thermal phase transition temperature. Furthermore, the thin plate-like crystal exhibited two-step bending motion by the photo-triggered phase transition and then the photoisomerization (Fig. 1c). Finally, by alternate irradiation of UV and visible light $(488 \mathrm{~nm})$ from the left, the plate-like crystal on the glass surface locomoted in the lower right direction (Fig. 1d). This finding leads to generalize the photo-triggered phase transition phenomenon and indicates that the photo-triggered phase transition enables to create various motions of crystals such as locomotion.
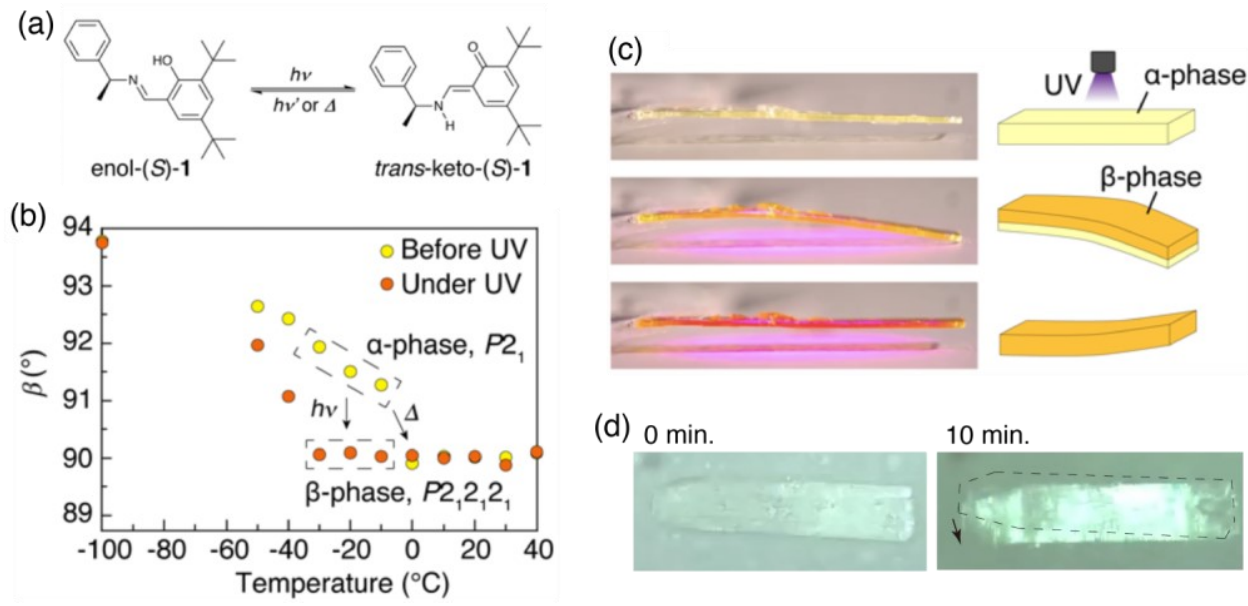

(d) 0 min. $10 \mathrm{~min}$.

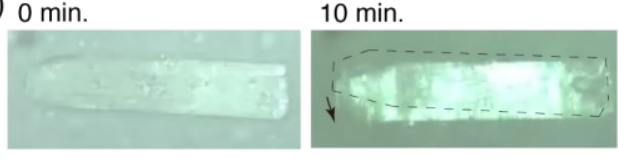

Figure 1 (a) Photoisomerization of enol- $(S)$-1. (b) Temperature dependence of the b-angle before and under UV light irradiation. (c) Two-step bending of the thin-plate crystal. (d) Locomotion of the crystal on the glass plate.

[1] Edited by H. Koshima. (2019) Mechanically Responsive Materials for Soft Robotics, Weinheim: Wiley-VCH.

[2] H. Koshima, N. Ojima \& H. Uchimoto. (2009) J. Am. Chem. Soc. 131, 6890-6891.

[3] T. Taniguchi, H. Sugiyama, H. Uekusa, M. Shiro, T. Asahi \& H. Koshima. (2018) Nat. Commun. 9, 538.

[4] Y. Hagiwara, T. Taniguchi, T. Asahi \& H. Koshima. (2020) J. Mater. Chem. C 8, 4876-4884.

[5] T. Taniguchi, H. Sato, Y. Hagiwara, T. Asahi \& H. Koshima. (2019) Commun. Chem. $2,19$.

[6] H. Koshima, R. Matsuo, M. Matsudomi, Y. Uemura \& M. Shiro. (2013) Cryst. Growth Des. 13, 4330-4337.

[7] A. Takanabe, T. Katsufuji, K. Johmoto, H. Uekusa, M. Shiro, H. Koshima \& T. Asahi. (2017) Crystals. 7, 7.

Keywords: mechanical crystals; locomotion; photo-triggered phase transition; salicylideneamine 